Maria do Rosário Ferreira

Coordenaçáo

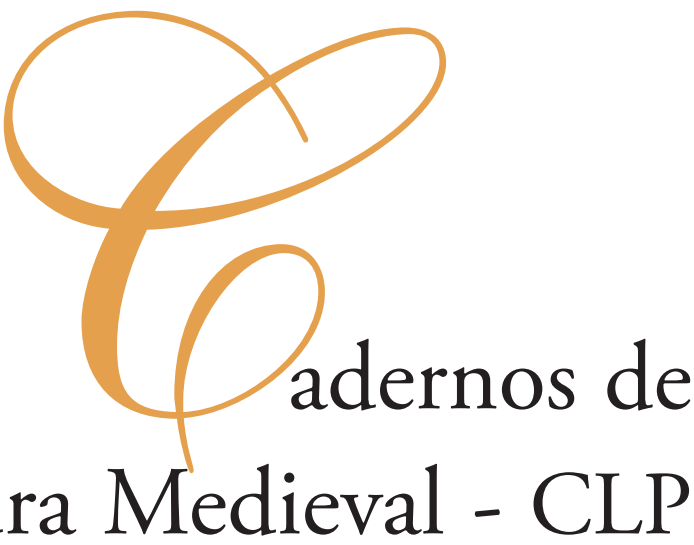

O Contexto Hispânico da Historiografia Portuguesa nos Séculos XIII e XIV

Em mémoria de Diego Catalán 
Inés Fernández-Ordóńez

Universidad Autónoma de Madrid

\section{DIEGO CATALÁN Y LA HISTORIOGRAFÍA MEDIEVAL HISPÁNICA}

Como alumna de Diego Catalán, tengo que agradecer de corazón que se dedique esta jornada a su memoria en la Universidad de Coimbra. Portugal fue siempre tierra muy grata para él, elegida con frecuencia para sus vacaciones, y en la que tuvo grandes amigos, como Luís F. Lindley Cintra. Ante la difícil tarea de sintetizar las principales aportaciones de Diego Catalán al estudio de la historiografía medieval hispánica, me acogeré al hilo histórico, al de la propia vida de Diego, para intentar comprender el nacimiento de su interés por la historiografía.

\section{Un interés temprano por la Historia}

Tras terminar los estudios de Filología Románica con sólo veinte años en 1949, Diego Catalán preparó su tesis bajo la dirección de Rafael Lapesa sobre la Crónica de Alfonso XI. Una redacción amplia desconocida, que defendió en 1951 (y que obtuvo el Premio Extraordinario en 1952). De ella saldrían dos libros, los dos primeros: Poema de Alfonso XI. Fuentes, dialecto, estilo (1953) y Un prosista anónimo del siglo XIV (La Gran Crónica de Alfonso XI. Hallazgo, estilo, reconstrucción) (1955). Aunque su interés por la historiografía sobre Alfonso XI surgió a partir del estudio de un romance histórico, El Prior de San Juan (según él mismo explica en su introducción a la Gran crónica de Alfonso XI ), la elección como tema de tesis de un texto cronístico (y no de una tradición poética o un tema de carácter lingüístico), creo que debe estimarse como prueba de la existencia en él de un interés decidido por la Historia, vocación tan poderosa al menos como la que sintió por

\footnotetext{
${ }^{1}$ Ed. Catalán (1977: 7-8).
} 
la Filología, y, desde luego, mucho mayor que la que sintió por la Lingüística, disciplina cuyo cultivo abandonó por completo desde mediados de los años setenta. Ese interés por la Historia (una vez me confió que en realidad hubiera querido estudiar Historia, y no Filología) impregna las tres líneas de investigación fundamentales a las que dedicó su atención, la historia de la lengua y la dialectología, la historiografía medieval y la épica y el romancero, y explica que en su persona se reúnan de forma totalmente inusual los conocimientos de un sabio historiador y los de un experto filólogo. La Historia como vía necesaria para la comprensión de los textos o de los comportamientos lingüísticos (y de la vida) y la Filología como herramienta imprescindible para la reconstrucción histórica son principios constantes que operan activamente en gran parte de su obra. La elección de un tema historiográfico para su tesis creo que cobra aún más relieve si se sopesa que durante los años de formación universitaria previa al doctorado (1944-49) las investigaciones en que trabajó, tutelado por las sabias guías de su abuelo, Ramón Menéndez Pidal, y del futuro director Lapesa, fueron de carácter lingüístico o romacerístico. En lo lingüístico estuvo dedicado al estudio del diminutivo en la toponimia, el límite de $f-h$ - en el oriente de Asturias y noreste de León, reelaboración de El dialecto leonés de Menéndez Pidal, índices de las voces estudiadas en Orígenes del español; en lo romancerístico, Catalán trabajó en cartografía romancística, en encuestas de campo de romances tradicionales, y en la reelaboración de los estudios de romances de tema histórico de Menéndez Pidal. Influido por los intereses de su abuelo, al que estuvo muy unido, y con su educación literalmente en manos de su familia, como resultado de la guerra, Diego Catalán se familiarizó desde muy joven con los campos de investigación de su abuelo. Así nos cuenta que «en los primeros ańos de Universidad sólo un par de profesores me proporcionaron algunos conocimientos de interés [...] En medio de la decepción de lo que podía recibir en los cursos universitarios, comencé, por invitación de mi abuelo, Ramón Menéndez Pidal, a manejar en casa, junto a materiales de interés lingüístico, otros pertenecientes a su archivo sobre el Romancero ${ }^{2}$. Pero pese a ello, eligió como tema de tesis uno de carácter historiográfico.

En realidad, creo que no es desacertado afirmar que la historiografía es el campo de investigación cultivado por Diego Catalán en que menos contó la herencia recibida de Menéndez Pidal y en el que sobresale el carácter pionero e innovador de su trabajo que, por lo general, se realizó en solitario -los equipos formados en ese terreno no son parangonables,

\footnotetext{
2 Catalán (2001: 262).
} 
en resultados, tamaño ni ligazón a los constituidos en el campo del romancero-. Tres son los aspectos generales que creo deben destacarse de su actividad. En primer lugar, su interés por la historiografía como tal, como textos de génesis literaria. A diferencia de Pidal, que estudió las crónicas medievales de forma subordinada a su valor testimonial para el conocimiento de la poesía tradicional, Catalán les prestó atención por sí mismas, como textos dignos de ser investigados como construcciones literarias que responden al entorno socio-político y cultural del que surgen. El papel central que la historiografía ha alcanzado últimamente dentro de la historia de nuestra literatura medieval se debe, en gran medida, a sus trabajos. En segundo lugar, se trata de un edificio construido sobre el ejercicio riguroso y meticuloso de la crítica textual, entonces apenas practicada en la Filología Hispánica. En general, toda su investigación parte del principio de no confundir texto con testimonio, principio que sus trabajos han contribuido a difundir de forma modélica. Así pudo desenmarañar tradiciones textuales complejísimas que le permitieron demostrar qué y qué no pertenecía a ciertas obras ya conocidas, al tiempo que identificaba otras hasta entonces desconocidas. En tercer lugar, sus trabajos comprenden un arco cronológico amplísimo y, como buen medievalista, sin límites establecidos por las lenguas, sea el latín o el romance, el gallego-portugués, el castellano, el navarro-aragonés o el catalán: así, sus estudios abarcan desde las primitivas crónicas asturianas hasta los textos hispánicos del siglo XV.

Diego Catalán llegó a conocer profundamente los problemas de un inmenso conjunto de obras historiográficas a partir del examen detenido de sus códices. El número de manuscritos de las mismas supera con gran frecuencia el de las obras literarias canonizadas en las historias de la literatura y nos da la medida de la popularidad y de la importancia que alcanzaron en su época. Pese a ello, las crónicas no suelen encontrar la acogida que merecen en las historias de literatura, quizá, cabría pensar, no sólo porque no se hayan valorado literariamente sino también porque el desbroce previo textual que exige su estudio riguroso requería una capacidad de trabajo que no está al alcance de la mayoría.

Por último, hay que valorar también en la preocupación de Diego Catalán por la historiografía su labor editorial, ya que gracias a él se publicaron no pocas obras historiográficas medievales en la colección del Seminario Menéndez Pidal, por él impulsada, «Fuentes cronísticas de la Historia de España», de la misma forma que impulsó las ediciones de colecciones y catálogos de romances.

La cantidad y calidad de los estudios de Diego Catalán sobre la historiografía medieval hispánica es tal que no es fácil compendiarlos. Aún así, pueden clasificarse en varios grandes campos: la historiografía escrita en torno a Alfonso XI, la historiografía relacionada 
con la Estoria de España de Alfonso X el Sabio, desde sus fuentes latinas hasta sus más tardías derivaciones medievales, la historiografía gallego-portuguesa, la historiografía navarra, y la historiografía vinculada a Rodrigo Jiménez de Rada. En todos ellos las aportaciones de Catalán parten del conocimiento directo de los numerosísimos manuscritos para construir una interpretación histórica y literaria de los textos estudiados.

\section{La historiografía en torno a Alfonso XI}

En su tesis ${ }^{3}$, en los libros antes citados, y luego en La tradición manuscrita en la "Crónica de Alfonso XI» (1974), veinticinco años posterior, Catalán pudo probar la existencia de dos versiones de la Crónica de Alfonso XI, la primera o Crónica de Alfonso XI, que fechó hacia 1344 y una refundida posteriormente, la Gran crónica de Alfonso XI, que editó (1977) y que pudo fechar a finales del siglo XIV. Gracias a Catalán conocemos la existencia de varias ramas textuales de la Crónica de Alfonso XI: la integrada dentro de la Crónica de cuatro reyes (Alfonso X, Sancho IV, Fernando IV y Alfonso XI), la vulgata, sobre la que partió la refundición de la Gran crónica, y aún otras posteriores fechadas en 1415 y 1489. Como parte fundamental de estos estudios imprescindibles sobre la historiografía en torno a Alfonso XI debe mencionarse su libro sobre el Poema de Alfonso XI: Fuentes, dialecto, estilo (1953), texto que pudo datar en 1348 y que estudió en relación con la Crónica y la Gran crónica.

Por desgracia, y aunque anunciaba en 1974 la edición de la Crónica de Alfonso XI, y posiblemente tuviera una edición en barbecho, ese texto nunca vio finalmente la luz. A día de hoy, seguimos a falta de esa edición, tan necesaria para el estudio de la historiografía del siglo XIV. Este campo sobre Alfonso XI, que gracias a las aportaciones de Diego fue roturado por vez primera y quedó preparado para su investigación ulterior, ha sido quizá el que menos ha atraído a los filólogos posteriores, que no han recogido la cosecha sembrada.

\section{La Estoria de España de Alfonso X}

Quizá la investigación historiográfica de Diego Catalán es sobre todo conocida y reconocida por su labor en torno a Alfonso X. El interés de Catalán por la Estoria

\footnotetext{
3 Catalán (1951, 1955b).
} 
de España de Alfonso X el Sabio nació de la lectura y reseña crítica (1959-60) de los primeros dos volúmenes de la edición de la Crónica geral de Espanha de 1344 de Luís Filipe Lindley Cintra (1951-90). A partir de ese estudio de Cintra, que revisaba en gran medida las conclusiones de Menéndez Pidal sobre las varias crónicas generales de España, Catalán inició una línea de investigación que lo acompañó hasta el final de su vida. Aunque sus descubrimientos no admiten fácil síntesis, es necesario explicar que el primero de ellos fue el carácter facticio del segundo de los manuscritos que Menéndez Pidal había utilizado como base de la edición de la Primera Crónica General, $\mathrm{E}_{2}$ (Biblioteca de San Lorenzo de El Escorial, X-I-4), en el cual se habían empalmado, a mediados del siglo XIV, el texto original alfonsí con una Versión amplificada de 1289, de época de Sancho IV, y con otros textos posteriores del siglo XIV, como la Crónica particular de San Fernando, que definió e identificó. El hallazgo, publicado en el libro De Alfonso X al conde de Barcelos (1962), supuso desligar la edición pidalina de la Primera crónica del texto genuino de la Estoria de España alfonsí, conservado en otros códices. Fue él el que devolvió también a la obra su denominación auténtica, Estoria de España, y la liberó de la inventada por Menéndez Pidal en su edición, Primera crónica general. A Catalán debemos asimismo la identificación y reconstrucción precisa, códice a códice, de las varias versiones de la Estoria de España, la concisa o primitiva, la crítica y la amplificada, y el estudio de los procedimientos que se siguieron para componerlas en el taller historiográfico alfonsí. Ya en ese libro de 1962 se apunta la idea de la existencia de una perdida Versión crítica de la Estoria de España de la que derivaban conjuntamente la Crónica general vulgata y la Crónica de veinte reyes, reconstrucción textual que vio confirmación veinte años después al aparecer, en 1983, un manuscrito que venía a hacer realidad lo antes supuesto. Ese trabajo de décadas sobre la Estoria de España culminó con la publicación de De la silva textual al taller historiográfico alfonsí (1997), que expone pormenorizadamente toda la tradición textual de la Estoria de España, desde el origen compositivo hasta la recepción de la obra en los dos siglos posteriores. A partir de su conocimiento de esas versiones, Catalán pudo identificar y realizar estudios detenidos de muchas obras derivadas, como la Crónica abreviada de don Juan Manuel, la Crónica fragmentaria, la Crónica de Castilla, la Crónica ocampiana o las Estorias del fecho de los godos del siglo XV. No menos importante es su reconstrucción de textos perdidos que vemos incorporados como fuente a otras obras, como es el caso de la Estoria caradignense del Cid, la Historia hasta 1288 dialogada, la Historia menos atajante sobre Alfonso VIII o la Estoria de los reyes moros de 
Sujulberto, obras hasta entonces completamente desconocidas y que hoy se estudian como tales. Por otro lado, el conocimiento profundo de los textos historiográficos le condujo ya entonces a revisar la metodología pidalina sobre qué aspectos debían juzgarse o no poéticos en las prosificaciones cronísticas. Así, por ejemplo, respecto al Mio Cid conservado en la Estoria de España o a las supuestas Mocedades de Rodrigo transmitidas por la Crónica de Castilla, Catalán pudo probar que la inventiva de los juglares no era mayor que la de los historiadores. Las conclusiones alcanzadas se recogen en La Estoria de España de Alfonso X: Creación y evolución (1992) (colección organizada de artículos previos sobre el tema publicados entre 1963 y 1977, más algunos otros), que dedicó a Cintra «por cuya amistad inicié mi actividad en este campo de trabajo».

\section{La historiografía gallego-portuguesa}

Fue, en efecto, a partir del estudio de Cintra cómo Catalán se sumergió en el océano historiográfico alfonsí y es por ello que ya en el libro de 1962 se realizan aportaciones de primer nivel para la historiografía portuguesa, en especial, en lo relativo a las fuentes manejadas por Pedro Afonso de Barcelos en la composición de la Crónica geral de 1344 . Entonces Catalán pudo probar la existencia y utilización de una perdida Crónica portuguesa de España y Portugal, que situó elaborada hacia 1340 en Portugal; asimismo estudió y descartó el entronque de la Versión gallego-portuguesa de la Estoria de España empleada por Barcelos con los códices de la misma conservados (con precisiones en 1992), frente a lo que había supuesto Cintra; y, finalmente, demostró el conocimiento de una versión refundida del Liber regum, caracterizada por haber interpolado la Leyenda de Bamba labrador y la materia de Bretaña a través del Brut de Wace, versión que bautizó Libro de las generaciones, localizó en Navarra y dató hacia 1260. Como complemento de enorme relevancia a estos hallazgos, Catalán editó, en colaboración con María Soledad de Andrés, la Crónica de 1344 (1971), en la parte inicial del texto, con el Libro de las generaciones, y, más tarde, otra de las fuentes de la Crónica de 1344, la Crónica del moro Rasis (1975), una traducción del texto árabe del historiador hispano-árabe al-Rāzī, que acompañó de un estudio memorable sobre la historia pre-islámica de España en las fuentes hispano-árabes de las que bebía al-Rāzī, estudio que los arabistas y estudiosos de Al-Andalus siguen manteniendo como referencia. 


\section{La historiografía Navarra}

La identificación de la versión del Liber regum empleado por don Pedro de Barcelos, a la que acabo de referirme el Libro de las generaciones, inició en Catalán una línea de investigación sobre ese texto que nunca articuló en un libro de conjunto, pero que bien podría constituirlo si se reunieran las notas que le dedicó en varios de sus trabajos.

Según demostró Diego Catalán, El Libro de las generaciones constituía el primer testimonio amplio de la difusión de la materia artúrica en la Península Ibérica y uno de los más antiguos, junto al Poema de Fernán González, para la leyenda de Bamba. En cuanto al Liber regum navarro original, el primer documento historiográfico y literario peninsular en romance, que puede definirse como una historia genealógica universal, Catalán ha aportado precisiones que pueden darse por definitivas. Aunque el códice en que se conserva no contiene el linaje del Cid, el autor muestra que esta importante sección perteneció desde un primer momento a la obra, y que es parte constitutiva de la misma y que debe datarse hacia 1194-96. También Catalán probó que hacia 1220 se realizó una versión castellana de este texto, actualizándolo de forma sucinta, versión que fue empleada por Rodrigo Jiménez de Rada en su Historia gothica. Catalán también ha señalado la utilización del Liber regum por el traductor de la obra de Jiménez de Rada que se conoce como Estoria de los godos hacia $1252-53$ y ha discutido las características de la versión que pudo ser empleada por la Estoria de España alfonsí y la *Crónica portuguesa de España y Portugal de hacia 1340, de apariencia similar. La llegada de la historiografía genealógica navarra a Portugal y el interés que suscitó, sustentan la hipótesis del liderazgo navarro en la construcción del discurso historiográfico peninsular.

\section{La Historia de Rebus Hispaniae de Rodrigo Jiménez de Rada}

Precisamente la obra historiográfica de un navarro, Rodrigo Jiménez de Rada, y sus derivaciones románicas fue el último objeto de investigación historiográfica de Catalán, tema sobre el que escribió, en colaboración con Enrique Jerez, y tras su jubilación, un libro monumental, «Rodericus» romanzado en los reinos de Aragón, Castilla y Navarra (2005). El libro transforma de forma radical el panorama crítico sobre los textos en romance derivados de De rebus Hispanie, como la Estoria de los godos y la Crónica de San Juan de la Peña en sus diversas versiones y es el digno colofón de toda una vida dedicada al estudio 
de la historiografía medieval. Tomando como punto de partida la tradición latina, en él se fija la existencia de dos versiones de De rebus Hispanie de Rodrigo Jiménez de Rada, que se datan en 1243 y 1246 , y se estudian todos los textos derivados en lengua romance entre los siglos XIII y XV. Entre las aportaciones del libro, debe destacarse, por su radical novedad, que Catalán pudo probar que la primera versión romance de De rebus fue la Estoria de los godos, elaborada hacia 1252-53 en el entorno de los señores de Albarracín, los Azagra, en conexión con el arzobispado de Toledo. No menos importante es que esa Estoria fue la base de gran parte de la historiografía navarra y aragonesa posterior: de la Crónica de 1305, de la Crònica real de Pedro IV (o Crónica de San Juan de la Peña), de la que fija y data las tres versiones consecutivas, y de las Canónicas de García de Euguí.

Una nueva valoración de la relación entre épica e historiografía

Por último, es necesario subrayar que el estudio contextualizado de los textos historiográficos condujo a Diego Catalán a una profunda revisión del estado de la cuestión heredado en torno a la épica española. Consciente de que los historiadores alteran sus fuentes de acuerdo con sus intereses y mentalidad, y conocedor como pocos del «lenguaje» de la literatura de transmisión oral, Diego estudió en La épica española: Nueva documentación y nueva evaluación (2000) el testimonio indirecto que sobre la épica española aportan las fuentes historiográficas, latinas y romances, para juzgar, con resultados asombrosos, qué puede estimarse de origen poético o no en ellas. Una de las grandes novedades de este libro es la superación de un viejo prejuicio de la escuela pidalina: el de creer en el valor «histórico» de la epopeya o de los relatos cronísticos. Si algo nos enseña Diego Catalán es que el valor «histórico» que debemos conceder a esos testimonios no es otro del que tienen como construcciones literarias al servicio de intereses o preocupaciones de personajes o grupos sociales, tal como muestra de forma magistral en su estudio sociopolítico del Poema de mio Cid o en la forma en que fue utilizado el personaje por la Crónica de Castilla (estudios que reunió con otros de sesgo puramente histórico sobre la Navarra del siglo XII en el libro El Cid en la historia y sus inventores, 2002).

\section{Un investigador sin par igual}

Parece imposible que tal cantidad de publicaciones de tan altísima calidad hayan podido ser acometidas por una sola persona, y que esa persona al mismo tiempo viviera a caballo de 
dos continentes y dirigiera simultáneamente dos centros de investigación, en Madrid y en California. La vitalidad, la energía y la capacidad de trabajo de Diego Catalán nunca tuvieron límites y no disminuyeron en lo más mínimo hasta el final de su vida. Llenos de datos, de documentación que sustenta lo afirmado y nos conduce, paso a paso, tras los razonamientos del autor, en una estructura siempre cuidadosamente ordenada y rotulada, carentes de retórica hueca, atiborrados de notas sustanciosas con información suplementaria, adicionados con índices de consulta por varios criterios que él mismo componía manualmente, no son los estudios de Diego Catalán fáciles de leer para el lector que no esté presto a estudiar. El rigor y la densidad de la investigación exigen atención y disposición al trabajo, aparte del interés que se le presupone al estudioso.

La autoexigencia y la curiosidad infinita que preside toda la actividad de Diego Catalán explican que todo su trabajo estuviera sometido a una autocrítica continua, con redacciones varias según pasaban los años, y que fuera capaz de renovar radicalmente tantos campos de investigación. Esa búsqueda de la verdad subyace al ejercicio crítico del trabajo ajeno pero, también y sobre todo, del propio. Como hemos visto antes, fue en una reseña crítica, publicada en 1959-60, de la edición de la Crónica de 1344 de Luís F. Lindley Cintra (1951-54, 1961), amigo y colega al que admiraba enormemente, cómo se gestó De Alfonso Xal conde de Barcelos (1962), libro que supuso el punto de partida de una revolución copernicana para la historiografía medieval derivada de la Estoria de España de Alfonso X. También en un ejercicio de autocrítica poco común, Diego Catalán mismo desmontó, veintitrés años después de presentarla, las conclusiones de su tesis sobre la Gran crónica de Alfonso XI (1951), texto que entonces había datado como anterior a la Crónica. La aparición de un nuevo manuscrito en los años sesenta, que estudió detenidamente, le condujo a demostrar, en su libro La transmisión manuscrita de la Crónica de Alfonso XI (1974), que la relación era exactamente la inversa de lo por él antes supuesto. En 1977, en la reimpresión de la edición de Menéndez Pidal de la Primera crónica general, se anuncia en la portada que irá acompañada de un «Estudio actualizador de Diego Catalán». Ese trabajo inédito, que no se publicó entonces, sin embargo existía en 1983, cuando Diego lo puso a nuestra disposición, sus alumnos historiográficos, como guía orientadora de nuestras tesinas y tesis: sólo vería la luz, radicalmente transformado, en 1997, veinte años después de ser anunciado, con el título de De la silva textual al taller historiográfico alfonsí. Lo que empezó en 1999 o quizá antes como un artículo que actualizase algunos aspectos de viejos trabajos sobre las versiones en romance del Toledano, a raíz de la aparición de la edición crítica del texto latino por Juan Fernández Valverde (1987), acabó por convertirse en el 
voluminoso «Rodericus» romanzado (2005). Este imperante deseo de alcanzar siempre el estado más avanzado y perfectible en la investigación de las cosas explica también que las diversas colecciones que recogieron sus artículos previos (en lingüística, historiografía o romancero) estén siempre revisadas por el autor, en la redacción y en muchos aspectos de contenido, con lo que, a menudo, se convierten en trabajos parcialmente nuevos: así sucede, por ejemplo, en La Estoria de España. Creación y evolución (1992) o en El Cid en la historia y sus inventores (2002). La existencia de investigaciones «latentes», en barbecho, que se estancaban o progresaban a lo largo de los años, en una tarea de renovación permanente, sacaba a la luz de repente estudios admirables, como el dedicado a las raíces históricas y la tipología en la tradición oral del romance de La muerte del príncipe don Juan, publicado en 1998, pero de cuya existencia yo había oído hablar desde principios de los años ochenta. En ese estado han debido quedar muchos trabajos, como la edición de la Crónica de Alfonso XI o una nueva edición de la Estoria de España (de la que venía hablando hace algunos años), y otros tantos materiales que necesitarían de alguien que los editase con el cuidado y la generosidad con que Diego Catalán editó los de su abuelo.

No puede sino dejarnos boquiabiertos que esa potencia creativa y esa disposición hercúlea hacia el trabajo no palidecieran en nada al final de su vida. Es más, fue precisamente en los años que siguieron a su jubilación en 1998 cuando Diego Catalán pudo dedicarse en cuerpo y alma a trabajos de envergadura colosal y que resulta difícil imaginar que hayan sido escritos por una persona en la década de sus setenta años. Fue justo entonces cuando terminó o escribió algunos de sus libros fundamentales como La épica española (2000), El Archivo del Romancero (2001), «Rodericus» romanzado (2005) y el libro -ya enviado a la imprenta cuando falleció- La enigmática carta del embajador (2008), volúmenes cualquiera de ellos que, aun aisladamente, nos requerirían a muchos una vida entera de dedicación.

Pero su vocación filológica no fue la del erudito solitario. Hay que destacar que Diego fue generoso con sus maestros, Menéndez Pidal y Lapesa, a los que organizó sendos homenajes, y también con sus alumnos, a los que supo interesar, ayudar y organizar en equipos de investigación.

Cuando en 1983, Diego ofreció a un conjunto de alumnos de la Universidad Autónoma de Madrid la posibilidad de trabajar en historiografía medieval, ello, en principio, nos asustó un poco, por ser un campo del que ignorábamos todo. Pero pronto el ofrecimiento se acompañó de los seminarios sobre historiografía medieval que comenzó a enseñar generosa y gratuitamente Diego en la antigua casa de Menéndez Pidal en el Olivar de 
Chamartín, de forma adicional a las clases regulares. Ello unido a la aparición en esa época de dos manuscritos desconocidos de la Estoria de España en Salamanca, cuyo estudio nos propuso Diego inmediatamente como tema de tesina, acabaron por decidirnos a unos pocos por el camino historiográfico.

Durante la redacción de la tesina y de la tesis, que en mi caso tuvo como arranque el estudio de uno de los manuscritos de Salamanca, esos alumnos «historiográficos» asistimos a varios seminarios y cursos de doctorado en la casa del Olivar de Chamartín. Allí nos enseñó Diego con paciencia ciertos rudimentos de paleografía que nos permitieron leer aquellos manuscritos medievales y puso a nuestra disposición todos los materiales necesarios: su «inédito de Chamartín»-que acabaría por convertirse en el libro La silva textual, de 1997-, reproducciones de manuscritos, entonces mucho más costosas y difíciles de conseguir que ahora, fuentes latinas -que también entonces manejábamos en las ediciones originales del siglo XVII y XVIII, allí conservadas, a falta de las ediciones modernas y críticas que después se han publicado-, e incluso alguna antigua transcripción que Tomás Navarro Tomás preparó para don Ramón. Con todo ello se aseguraba que pudiéramos tener en la mano, sin esfuerzo alguno, toda la necesaria información documental y bibliográfica. También se ocupó de obtener una pequeña ayuda económica, solicitada a la Caja de Ahorros de Salamanca, propietaria de los códices, para que nuestra dedicación a la investigación tuviera cierta financiación durante aquel curso. Como un verdadero «taller historiográfico»-o "Laboratorio historiográfico», tal como figuraba en la puerta en un cartel de letra de su madre, Jimena Menéndez Pidal- varios alumnos llegamos a defender tesis sobre nuestras crónicas medievales y contribuimos modestamente a «descifrar»el complejo panorama de la tradición textual de la Estoria de España. Si ello fue posible, se debe precisamente a la capacidad de Diego de organizar equipos de investigación en torno a un campo (téngase en cuenta que cada una de las tesis entonces planteadas suplementaba a las demás), método colaborativo y colectivo que había desarrollado con resultados modélicos en el terreno del romancero. Y esa capacidad radicaba no sólo en su sabiduría sino en su personalidad carismática, que le hacía centro de las reuniones y de las conversaciones -fuera en la casa del Olivar de Chamartín hablando de un tema erudito, fuera en la mesa de un restaurante conversando de política-. Diego «desordenaba el aire» allí donde estaba. Aunque Catalán afirmó en el prólogo de su libro aún inédito la La enigmática carta (2008) que «No reconozco a nadie como discípulo mío. No soy un cristiano de la Ciencia filológica. Como los beduinos, siembro de paso y levanto mi tienda», lo cierto es que supo enseñar mucho y a muchos. 
Poseedor de conocimientos profundos en diversas disciplinas, crítica textual y herramientas filológicas, crítica literaria, lingüística e historia, Diego Catalán supo combinarlas para levantar edificios con resistencia sísmica, tal es la multiplicidad y firmeza de sus cimientos. Cuando sus conocimientos se aplican de forma transversal a varias disciplinas, la perspectiva plural produce hallazgos deslumbrantes y suele haber un denominador común: la Historia. Diego Catalán fue, ante todo, un historiador de textos, que supo descifrarlos magistralmente con las herramientas de la Filología (la lingüística, la crítica literaria y textual) y de la Historia, y que, al tiempo, supo extraer de ellos su valor como testimonios (de mentalidades, situaciones, hechos o individuos) históricos (véase el prólogo memorable que escribió en 1982 para la reedición de Los españoles en la historia de Menéndez Pidal). En el prólogo de su último libro, La enigmática carta del embajador, 28 de mayo/6 de junio de 1562, aún inédito, como si intuyera que este podría ser su testamento intelectual, habla con franqueza inusitada de su trabajo y confiesa: «Me considero un Filólogo... con inclinación a la Historia»; Dice: «No soy un "Historiador", ni intento serlo, porque (y hablo ahora en términos generales) no creo en la existencia de realidades "objetivas", reconstruibles a partir de lo documentado. Lo que "fue" no está constituido por "hechos" que sean, de por sí, significativos. El "significado" se lo dan los relatos en que los detalles documentados vienen a ser integrados. $\mathrm{Y}$ es preciso tener bien presente que todo relato es una narración, una ordenación creada por alguien y para algo. El caos de los hechos que se dieron en un determinado espacio temporal requiere la criba y la articulación de una mente interpretativa y expositiva para que cobre sentido» ${ }^{4}$.

Gracias a su mente interpretativa y expositiva de Historiador, nos ha legado una obra inmensa, única por su originalidad y de valor incalculable para la historia de la lengua, la literatura y la cultura españolas, que por sí misma merece un puesto de honor equiparable al de sus maestros Ramón Menéndez Pidal y Rafael Lapesa.

${ }^{4}$ 13/02/2008, http://olivarchamartin.blogia.com/2008/021001-prologo-al cancionero-en-cifra-de-perrenot.php. 


\section{Obras citadas de Diego Catalán Menéndez-Pidal:}

Catalán, Diego (1951). «Cronica de Alfonso XI»: una redacción amplia desconocida. Tesis doctoral. Universidad de Madrid.

- (1953). Poema de Alfonso XI. Fuentes, dialecto, estilo. Madrid: Gredos.

- (1955). Un prosista anónimo del siglo XIV (La Gran Crónica de Alfonso XI. Hallazgo, estilo, reconstrucción). La Laguna: Universidad de La Laguna.

- (1959-60). «La versión portuguesa de la Crónica General (Sobre L.F. Lindley Cintra, Crónica Geral de Espanha de 1344. Edição crítica do testo portugués, Lisboa 1951-54)». Romance Philology 13, pp. 363-72.

- (1962). De Alfonso X al Conde de Barcelos. Cuatro estudios sobre el nacimiento de la historiografía romance en Castilla y Portugal. Madrid: Seminario Menéndez Pidal/Gredos.

- y María Soledad de Andrés (1971). Crónica de 1344 que ordenó el Conde de Barcelos don Pedro Alfonso (estudio histórico y edición crítica), vol. I. Madrid: Seminario Menéndez Pidal/Gredos.

- (1974). La tradición manuscrita de la Crónica de Alfonso XI. Madrid: Gredos.

— y María Soledad de Andrés (1975). Crónica del moro Rasis. Versión del Ajbar muluk al-Andalus de Ahmad ibn Muhammad Mùsà al-Rāzì: romanzada para el rey don Dionis de Portugal hacia 1300 por Mohamed, alarife, y Gil Pérez, clérigo de Perianes Porcel (edición con la colaboración de Margarita Estarellas, Mercedes García Arenal y Paloma Montero). Madrid: Seminario Menéndez Pidal/Gredos.

- (1976). Gran crónica de Alfonso XI. Edición crítica y estudio. 2 voll., Madrid: Seminario Menéndez Pidal/ Gredos.

— (1982). «España en su historiografía: de objeto a sujeto de la historia». En: Ramón Menéndez Pidal. Los españoles en su historia. Madrid: Espasa-Calpe.

- (1992). La Estoria de España de Alfonso X. Creación y evolución. Madrid: Seminario Menéndez Pidal, Fundación Ramón Menéndez Pidal/Universidad Autónoma de Madrid.

- (1997). De la silva textual al taller historiográfico alfonsí. Códices, crónicas, versiones y cuadernos de trabajo. Madrid: Seminario Menéndez Pidal, Fundación Ramón Menéndez Pidal/Universidad Autónoma de Madrid

— (2000). La épica española. Nueva documentación y nueva evaluación. Madrid: Fundación Ramón Menéndez Pidal/Seminario Menéndez Pidal.

- (2001). El Archivo del Romancero: Patrimonio de la humanidad - Historia documentada de un siglo de historia-.

2 voll., Madrid: Fundación Ramón Menéndez Pidal/Seminario Menéndez Pidal.

- (2002). El Cid en la historia y sus inventores. Madrid: Fundación Ramón Menéndez Pidal.

- (2005). "Rodericus» romanzado en los reinos de Aragón, Castilla y Navarra (con la colaboración de Enrique Jerez). Madrid: Fundación Ramón Menéndez Pidal. 
- (en prensa). La enigmática carta del embajador, 28 de mayo/6 de junio de 1562. URL: http://olivarchamartin. blogia.com/2008/021301-indice-del-libro-la-enigmatica-carta-del-embajador-28-de-mayo-6-de-juniode-1562.php.

«Fuentes cronísticas de la Historia de España», colección dirigida por Diego Catalán:

I. Primera Crónica General de España por Alfonso X el Sabio; editada por Ramón Menéndez Pidal, con un estudio actualizador de Diego Catalán... 2 voll., Madrid: Seminario Menéndez Pidal/Universidad Complutense/Gredos, 1977.

II. Edición crítica del texto español de la Crónica de 1344, que ordenó el Conde de Barcelona don Pedro Alfonso. Preparada por Diego Catalán y María Soledad de Andrés, Madrid: Gredos/Seminario Menéndez Pidal, 1971.

III. Crónica del moro Rasis, versión del ajbār mulūk al-andalus de ahmad ibn muhammad ibn mūsà al-Rāzī, 889-955; romanzada para el rey don dionis de portugal hacia 1300 por mahomad, alarife, y gil pérez, clérigo de don perianes porçel. Diego Catálán y M. a Soledad de Andrés, con la colaboración de Margarita Estarellas, Mercedes García Arenal y Paloma Montero, prepararon esta edición pluritextual en el Seminario Menéndez Pidal, año 1974. Madrid: Gredos/Seminario Menéndez Pidal, 1975.

IV. Gran crónica de Alfonso XI, preparada por Diego Catalán. 2 voll., Madrid: Gredos/Seminario Menéndez Pidal, 1977.

V. La Estoria de España de Alfonso X. Creación y evolución, por Diego Catalán, Madrid: Fundación Ramón Menéndez Pidal/Universidad Autónoma de Madrid, 1992.

VI. Versión crítica de la Estoria de España. Estudio y edición desde Pelayo hasta Ordoño II, por Inés Fernández-Ordóñez, Madrid: Fundación Ramón Menéndez Pidal/Universidad Autónoma de Madrid, 1993.

VII. Las leyendas de los Señores de Vizcaya y la tradición melusiniana, por José Ramón Prieto Lasa, Madrid: Fundación Ramón Menéndez Pidal/Universidad Autónoma de Madrid/Universidad del País Vasco, 1995.

VIII. La Historia, creación literaria. El ejemplo del Cuatrocientos, por Cruz Montero Garrido, Madrid: Fundación Ramón Menéndez Pidal/Universidad Autónoma de Madrid, 1995.

IX. De la silva textual al taller historiográfico alfonsi. Códices, crónicas, versiones y cuadernos de trabajo, por Diego Catalán, Madrid: Fundación Ramón Menéndez Pidal/Universidad Autónoma de Madrid, 1997.

X. «Rodericus» romanzado en los reinos de Aragón, Castilla y Navarra, por Diego Catalán con la colaboración de Enrique Jerez, Madrid: Fundación Ramón Menéndez Pidal, 2005. 